\title{
PARASITISMO POR Dioctophyme renale ENCONTRADO EM CÃES DE ABRIGOS DO MUNICÍPIO DE SÃO MATEUS DO SUL, ESTADO DO PARANÁ
}

\author{
(Parasitism by Dioctophyme renale found in shelter dogs from municipality of São \\ Mateus do Sul, Paraná State) \\ Camila Tais Zanchett ${ }^{\star}$, Daniela Pedrassani \\ Universidade do Contestado
}

E-mail para contato: camilazanchett@outlook.com

\begin{abstract}
RESUMO - Dioctophyme renale é um nematódeo zoonótico que parasita os rins de carnívoros domésticos e silvestres no Brasil. Entre os cães têm maior prevalência em errantes, principalmente, por conta dos hábitos menos seletivos, tornando interessante o estudo com os mesmos. Animais que vivem em abrigos, geralmente apresentam histórico de viver em ambiente precário, acesso restrito à água limpa e alimento adequado e, em superpopulação, favorecendo a disseminação da dioctofimose. Por conta disso, este trabalho teve o objetivo de verificar a ocorrência do parasitismo por $D$. renale em cães de abrigos do município de São Mateus do Sul, estado do Paraná. Os animais foram transportados por uma Organização Não Governamental (ONG) até o Hospital Veterinário Escola para procedimento de ovariosalpingohisterectomia (OSH). Os resultados das análises hematológica, bioquímica e da urina foram comparados entre cães parasitados e não parasitados pelo teste $t$ de Student e a idade foi avaliada pelo teste exato de Fisher $(p \leq 0,05)$. Entre agosto e novembro de 2018, o abrigo encaminhou 38 fêmeas, sem raça definida para $\mathrm{OSH}$ e destas, três $(7,89 \%)$ apresentaram ovos do parasito na urina. Um, dentre os três cães positivos para $D$. renale foi a óbito durante a cirurgia e na necropsia uma fêmea do parasito foi observada no rim esquerdo. A creatinina foi significativamente mais alta nos cães que apresentaram ovos do parasito na urina $(p=0,015)$. Não foram observadas diferenças entre cães que apresentaram ou não ovos do parasita na urina em relação aos fatores: dosagem de ureia e de proteínas plasmáticas totais, hemograma, leucograma, análise física da urina, assim como idade $(p>0,05)$. É possível concluir que em cães do município, principalmente, os de abrigos, os exames rotineiros de urinálise e dosagem de creatinina, mesmo naqueles aparentemente saudáveis, são importantes para um diagnóstico precoce de parasitismo por $D$. renale.
\end{abstract}

Palavras-chave: canídeo; dioctofimose; nematódeo; rim; urinálise.

ABSTRACT - Dioctophyme renale is a zoonotic nematode that infects the kidneys of domestic and wild carnivores in Brazil. Among the dogs, it is highly prevalent in stray animals, mainly because of their less selective habits, making interesting the study with them. Shelter animals usually have a life background in a precarious and overpopulated environment with low access to good quality feed and water, which contributes to the spread of dioctophymosis. The objective of this work was to verify the occurrence of $D$. renale parasitism in shelters dogs from municipality of São Mateus do Sul, Paraná. Animals were transported by a non-governmental organization (NGO) to the Veterinary School Hospital for the procedure of total hysterectomy with bilateral salpingo-oophorectomy (HSO). The results of 
hematological, biochemical and urine analyses of parasitized and not parasitized dogs were compared using the Student t-test as well as age was evaluated by Fisher's exact test $(p \leq 0.05)$. From August to November 2018, the shelter sent 38 mixed-breed female dogs for HSO, and three $(7,89 \%)$ of them presented eggs of the parasite in the urine. One of these three positive dogs died during the HSO procedure. A female of $D$. renale was found in the left kidney over the necropsy. Creatinine was significantly higher for those dogs presenting eggs of $D$. renale in the urine $(p=0.015)$. No differences were found for dogs with or without parasite eggs in the urine for the following factors: urea and total protein test, hemogram, leukogram, urinalysis, as well as age ( $p>0.05)$. It is possible to conclude that in dogs from this municipality, especially in shelter dogs, routine urinalysis and creatinine dosage levels, even for those apparently healthy, are important for an early diagnosis of parasitism by $D$. renale.

Keywords: canids; dioctophymosis; nematode; kidney; urinalysis.

\section{INTRODUÇÃO}

O Dioctophyme renale, causador da dioctofimose, é o maior nematódeo conhecido de animais domésticos (Silveira et al., 2015; Lima et al., 2016). Apresenta distribuição mundial e é comumente descrito parasitando carnívoros domésticos e silvestres os quais atuam como seus hospedeiros definitivos. Além da importância em sanidade animal é de interesse em saúde pública, pelo seu caráter zoonótico (Kano, 2003; Yang et al., 2019).

Os ovos desse nematódeo são eliminados com a urina do hospedeiro definitivo e evoluem no meio externo, em locais de água doce até a formação da larva de primeiro estádio dentro do ovo, o qual é ingerido por hospedeiros intermediários, anelídeos aquáticos, nos quais se desenvolve a forma infectante (larva de terceiro estádio). Os animais e humanos adquirem a infecção a partir da ingestão dos hospedeiros intermediários $(\mathrm{HI})$ (anelídeos aquáticos da espécie Lumbriculus variegatus) ou de hospedeiros paratênicos (HP) (peixes e anfíbios) infectados com a forma infectante (Measures; Anderson, 1985).

Dentre os animais domésticos, o $D$. renale é observado com maior frequência em cães, principalmente, os errantes e semi-domiciliados, os quais, muitas vezes não tem acompanhamento periódico de um médico veterinário o que dificulta 0 diagnóstico do parasitismo (Kommers; Ilha; Barros, 1999). Os mesmos, possuem maior chance de se infectar através da ingestão acidental de anelídeos $(\mathrm{HI})$ juntamente com água de valetas e banhados ou pelo consumo de anfíbios ou peixes crus (HP) (Giorello et al., 2017; Pedrassani et al., 2017). A importância dos hospedeiros paratênicos na transmissão de $D$. renale para os cães requer maiores estudos, pois os HP apresentam um pequeno número de larvas do parasito em seus tecidos e em relação aos peixes, inclui a dificuldade de captura dos mesmos (Pedrassani et al., 2017; Mascarenhas et al., 2019).

A dioctofimose canina tem sido relatada, principalmente, na região sul do país, em Santa Catarina (Pedrassani et al., 2014; Rocha, 2017; Pedrassani et al., 2017), Rio Grande do Sul (Colpo et al., 2007; Silveira et al., 2015; Rappeti et al., 2017) e Paraná (Kano et al., 2003).

Animais que vivem em abrigos, geralmente, apresentam histórico de viver em ambiente precário, pouco acesso à água e alimento adequado e em superpopulação, assim, favorecendo a disseminação da dioctofimose. Levando em 
consideração a presença de um ambiente favorável ao parasitismo, este trabalho teve como objetivo averiguar a ocorrência de Dioctophyme renale em cães de abrigos do município de São Mateus do Sul, Paraná por meio de análise de urina e verificar se análises bioquímicas e hematológicas mostravam alterações decorrentes do parasitismo.

\section{MATERIAL E MÉTODOS \\ Amostragem e local de estudo}

Entre agosto e novembro de 2018 foi analisada uma amostragem não probabilística por conveniência, composta de 38 cadelas, sem raça definida, com idade média de 3 anos ( $\pm 1,5$ anos) encaminhados por uma Organização Não Governamental (ONG) do Município de São Mateus do Sul, Paraná. A ONG não possui sede, desse modo os cães que estão sob sua tutela são mantidos em residências de diversos voluntários aguardando adoção.

O município de São Mateus do Sul é cortado pelos rios Iguaçu e Potinga, e banhado ao sul pelo rio Negro, na fronteira com Santa Catarina (IBGE, 2018). O rio Iguaçu é dividido em baixo, médio e alto Iguaçu e a área média corta o município de São Mateus do Sul. Segundo Secretaria de Estado do Meio Ambiente e Recursos Hídricos (SEMA, 2010), esta área média, apresenta potencial contaminante e baixa infraestrutura de esgotos e drenagem. A ONG não forneceu a localização das residências onde os cães estavam abrigados, mas afirmou serem próximas a rios ou córregos d'água.

Os cães foram atendidos para procedimento cirúrgico de ovariosalpingohisterectomia (OSH) no Hospital Veterinário da Universidade do Contestado (UnC), Campus Canoinhas, sendo submetidos previamente a exame físico.

\section{Obtenção das amostras de sangue}

A coleta de sangue total foi realizada no momento dos exames préoperatórios, via punção da veia jugular, sendo coletados $5 \mathrm{~mL}$, com os animais contidos fisicamente. Metade do volume de sangue coletado foi acondicionado em tubo com EDTA para realização do hemograma e a outra metade foi acondicionado em tubos com ativador de coágulo e, em seguida, foi centrifugado a $460 \times \mathrm{g}$, por 10 minutos para obtenção do soro para os exames bioquímicos.

Hemograma: a contagem total de eritrócitos e de leucócitos foi realizada seguindo as recomendações de Weiser (2015) e os resultados comparados com o valor de referência dos parâmetros analisados para a espécie canina (Tabela 2).

Determinação da ureia e creatinina: para determinar os níveis de creatinina e ureia foram utilizados kits comerciais, sendo realizado conforme recomendações e métodos estabelecidos pelo fabricante. A leitura foi realizada em um analisador bioquímico semiautomático. Os resultados foram comparados com os valores de referência descritos por Bush (2004) (Tabela 2).

\section{Obtenção das amostras de urina}

A urina foi obtida por micção espontânea ou, como eram fêmeas, por compressão da vesícula urinária durante procedimento cirúrgico. Foram coletados no mínimo $10 \mathrm{~mL}$ de urina de cada animal e processada para urinálise e para pesquisa de ovos do parasito. 
Urinálise: a análise de urina foi realizada utilizando tiras reagentes comerciais seguindo as recomendações do fabricante. Os resultados foram comparados com os valores de referência dos parâmetros analisados para a espécie canina descritos por Kerr (2003), Bush (2004) e Sink e Feldman (2006) (Tabela 1).

Tabela 1 - Parâmetros analisados e valores de referência da urinálise realizada pelo método da fita reagente com amostras de urina dos cães da ONG submetidos à $\mathrm{OSH}$.

\begin{tabular}{cc}
\hline Parâmetro & Valor de referência \\
\hline Leucócitos & Negativo *** \\
Nitrato & Negativo * \\
Urobilinogênio $(\mu \mathrm{mol} / \mathrm{L})$ & $8 \mathrm{a} 17^{*}$ \\
Proteína $(\mathrm{g} / \mathrm{L})$ & $\leq 0,5^{*}$ \\
$\mathrm{pH}$ & $5,5 \mathrm{a} 7^{*}$ \\
Sangue (Células $\times 10^{3}$ hemácias $\left./ \mathrm{mL}\right)$ & $5 \mathrm{a} 15^{*}$ \\
Densidade & $1.015 \mathrm{a} 1.045^{* *}$ \\
Cetonas & Negativo * \\
Bilirrubina & $\leq+{ }^{*}$ \\
Glicose & Negativo ***
\end{tabular}

Fonte: * BUSH (2004); ${ }^{* \star}$ KERR (2003); ${ }^{* * *}$ SINK; FELDMAN (2006).

Pesquisa de ovos de Dioctophyme renale: a sedimentação foi feita por centrifugação de $10 \mathrm{~mL}$ de urina por 15 minutos a $460 \times$ g. Posteriormente, o sobrenadante foi desprezado e o sedimento ressuspenso no líquido restante para leitura em microscopia óptica de luz branca nos aumentos de 160 e 640 vezes (Sloss et al., 1999).

\section{Análise dos dados}

Tratou-se de um estudo do tipo transversal, com a prevalência calculada conforme Pereira (2008), no qual os resultados do hemograma, urinálise, ureia e creatinina foram comparados por Teste $t$ de Student e a associação entre a idade estimada do cão e a presença de parasitismo foi avaliada por Teste exato de Fisher. Em ambos os resultados foram considerados significativos quando $p \leq 0,05$.

\section{RESULTADOS}

No exame físico prévio ao procedimento cirúrgico nenhum dos 38 cães apresentou sinais clínicos sugestivos da parasitose. Porém, três fêmeas examinadas $(7,89 \% ; n=3 / 38)$ foram positivas pela detecção de ovos de $D$. renale no sedimento urinário (Figura 1a). Durante o procedimento de OSH, os trinta e oitos animais tiveram a cavidade abdominal e os rins inspecionados. Não foram observadas alterações morfológicas nos rins de 37 animais, porém, uma fêmea $(n=1 / 38)$, que foi positiva também pela presença dos ovos no sedimento urinário, apresentou a morfologia do rim esquerdo alterado, sugestivo para presença de $D$. renale.

Esse animal veio a óbito por parada cardiorrespiratória no transoperatório da $\mathrm{OSH}, \mathrm{e}$, na necropsia foi observado o rim esquerdo com perda da morfologia normal apresentando-se como uma bolsa com conteúdo no interior (Figura 1b), macio na palpação e com neovascularização. Após incisão desse rim, foi removida uma fêmea de $D$. renale de $51 \mathrm{~cm}$ de comprimento e observou-se apenas a cápsula renal com destruição total do parênquima (Figura $1 \mathrm{~b}$ - seta amarela). O rim direito 
apresentava-se com arquitetura preservada, porém, hipertrofiado (apresentando quase $1 / 3$ a mais do que o tamanho normal de um rim) (Figura $1 b$ - seta branca).
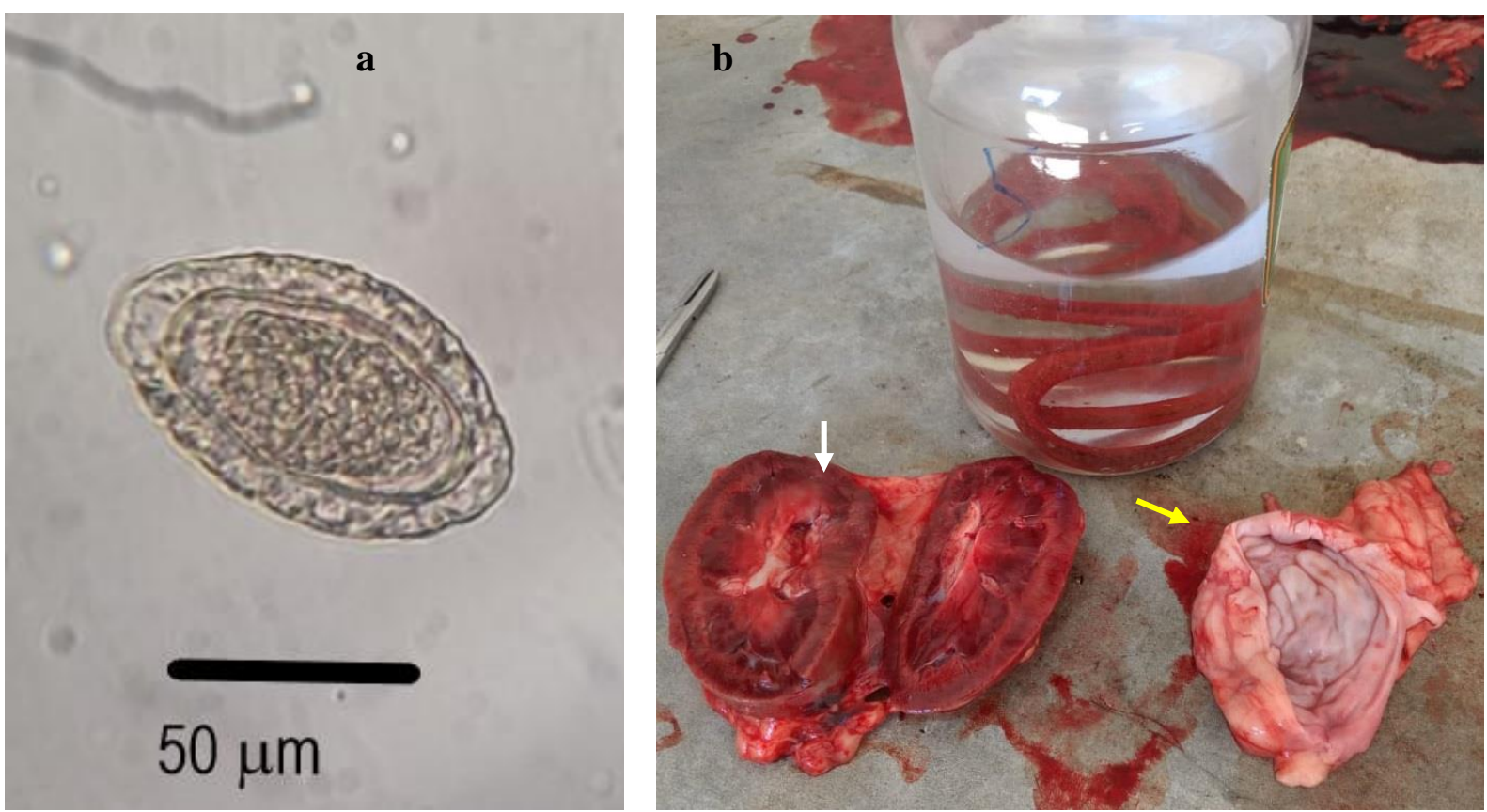

Figura 1 - Detecção do parasitismo por Dioctophyme renale. a) Ovo do parasito observado em três cães fêmeas pelo exame de sedimentação urinária. b) Rim direito (seta branca) e esquerdo (seta amarela) retirados da fêmea que veio a óbito durante o procedimento de OSH. É possível observar o rim direito não parasitado e o rim esquerdo onde uma fêmea do parasito (acondicionado no recipiente) foi encontrada.

Quanto à mensuração da creatinina sérica, os cães parasitados apresentaram aumento significativo dos níveis sanguíneos quando comparados aos não parasitados $(p=0,015)$ (Tabela 2). Os três cães positivos apresentaram hiperproteinemia e também 26, dentre os 35 não parasitados, apresentaram esta alteração. Os demais animais apresentaram normalidade para esse parâmetro.

Os animais positivos apresentaram idade estimada (conforme avaliação dentária) superior a três anos e nenhum dos cães do estudo apresentou valores abaixo do normal para a espécie em relação aos parâmetros do hemograma (Tabela 2). Apenas três animais apresentaram leucocitose e, destes, um estava parasitado por $D$. renale (diagnosticado pela presença de ovos na urina).

Não foram observadas diferenças estatísticas significativas entre cães que apresentaram ou não ovos do parasita na urina em relação aos fatores: dosagem de ureia e de proteínas plasmáticas totais (PPT), hemograma, leucograma, análise física da urina $(\mathrm{p}<0,05)($ Tabela 2$)$ assim como idade. 
Tabela 2 - Resultados de hemograma, leucograma, urinálise e análise de bioquímica renal de cães parasitados $(n=3)$ e não parasitados $(n=35)$ por Dioctophyme renale.

\begin{tabular}{|c|c|c|c|c|}
\hline \multicolumn{5}{|c|}{ Hemograma e Leucograma } \\
\hline Parâmetro & Valor referência ${ }^{a}$ & Parasitado* & $\begin{array}{c}\text { Não } \\
\text { Parasitado* }^{*}\end{array}$ & $\begin{array}{c}\text { Valor de } \\
\text { P }\end{array}$ \\
\hline Hemácias & 5,5 a 8,5 (Células x 1012/L) & $6,53 \pm 1,16$ & $5,88 \pm 1,41$ & 0,180 \\
\hline Hematócrito & 37 a $55 \%$ & $52 \pm 2$ & $50,97 \pm 7,04$ & 0,804 \\
\hline Leucócitos & 6 a 17 (Células x 103/UI) & $15183 \pm 4086,7$ & $12087 \pm 4476,8$ & 0,255 \\
\hline PPT & 5,7 a $7,7(\mathrm{~d} / \mathrm{dL})$ & $9,2 \pm 0,43$ & $8,54 \pm 1,18$ & 0,356 \\
\hline \multicolumn{5}{|c|}{ Urinálise } \\
\hline Parâmetro & Valor referência & Parasitado* & $\begin{array}{c}\text { Não } \\
\text { Parasitado* }\end{array}$ & $\begin{array}{c}\text { Valor de } \\
\text { P }\end{array}$ \\
\hline Proteína & $\geq 0,5(\mathrm{~g} / \mathrm{L})^{a}$ & $10,66 \pm 16,74$ & $23,4 \pm 34,23$ & 0,243 \\
\hline $\mathrm{pH}$ & 5,5 a $7^{a}$ & $6,16 \pm 0,28$ & $6,46 \pm 0,52$ & 0,345 \\
\hline Densidade & 1.015 a $1.045^{b}$ & $1025 \pm 0$ & $1021 \pm 5,66$ & 0,234 \\
\hline Glicose & Negativo ${ }^{c}$ & $1 \pm 0$ & $1 \pm 0$ & 0,774 \\
\hline \multicolumn{5}{|c|}{ Bioquímica renal } \\
\hline Parâmetro & Valor referência ${ }^{a}$ & Parasitado* & $\begin{array}{c}\text { Não } \\
\text { Parasitado* }\end{array}$ & $\begin{array}{c}\text { Valor de } \\
\text { P }\end{array}$ \\
\hline Creatinina & 0,5 a 1,5 (mg/dL) & $1,36 \pm 0,23$ & $0,99 \pm 0,24$ & $0,015^{\star}$ \\
\hline Ureia & 15 a $40(\mathrm{mg} / \mathrm{dL})$ & $35,66 \pm 13,27$ & $42,06 \pm 29,15$ & 0,712 \\
\hline
\end{tabular}

Fonte: a) Bush (2004); b) Kerr (2003); c) Sink; Feldman (2006). * significativo estatisticamente Legenda: PPT - Proteína plasmática total; *Média \pm Desvio padrão.

\section{DISCUSSÃO}

A partir das amostras de urina analisadas, foi possível detectar três casos de parasitismo por $D$. renale. Entretanto, como são animais recolhidos em situação de rua/abandono e sobre os quais se desconhece a procedência é difícil analisar dados relacionados às possíveis fontes de infecção no local de residência. Mas, é possível inferir que tenham enfrentado fome e sede e podem ter ingerido sapos, rãs e peixes (HPs), bem como água em valetas onde o anelídeo Lumbriculus variegatus $(\mathrm{HI})$ pode estar presente (Measures, 2001). A região parece ser favorável ao desenvolvimento deste parasito, uma vez que, nos munícipios catarinenses de Canoinhas (distante $17 \mathrm{Km}$ de São Mateus do Sul) e Três Barras (faz divisa com São Mateus do Sul) há relatos da ocorrência da forma adulta do $D$. renale em cães (Pedrassani et al., 2010; 2017) e gatos (Pedrassani et al., 2014) e de larvas em sapos (Pedrassani et al., 2009).

Em dois dos animais em que ovos do parasito foram visualizados na urina não foram detectadas alterações na arquitetura renal na inspeção visual durante a OSH. Isso pode ser decorrente de um parasitismo recente e no qual ainda há um pequeno grau de destruição do parênquima renal (Silveira et al., 2015). Após o diagnóstico de parasitismo, a ONG responsável foi informada que os animais estavam parasitados e que o tratamento de escolha era o cirúrgico (Secchi et al., 2010; Souza et al., 2019).

No terceiro animal parasitado, que veio a óbito no transoperatório da $\mathrm{OSH}$, foi observado na necropsia uma fêmea do parasito que foi encontrada dentro do rim esquerdo. Em relatos de literatura, o parasitismo é mais comum no rim direito, provavelmente devido a sua proximidade anatômica com o estômago e duodeno, 
que são os locais por onde a fase larval penetra para acessar a cavidade abdominal (Measures, 2001; Monteiro et al., 2002). Parasitos adultos podem ser observados no rim esquerdo quando larvas infectantes perfuram o estômago na grande curvatura para chegar à cavidade abdominal (Measures, 2001; Monteiro et al., 2002; Kano et al., 2003; Pedrassani et al., 2010). Na literatura existem poucos relatos com o diagnóstico do parasitismo no rim esquerdo (Li et al., 2010; Pedrassani et al., 2010; Perera et al., 2017).

O fato da ocorrência de $D$. renale em cães com diferentes idades indica que os cães podem já ter se infectado quando jovens (Pedrassani et al., 2017), permanecendo parasitados ao longo da vida, pois o período pré-patente e patente desse parasito são de, aproximadamente, 135 dias e 3 a 5 anos, respectivamente, se não diagnosticado previamente (Measures, 2001). Pedrozo et al. (2013) e Silveira et al. (2015) citaram que este parasito pode ser observado, principalmente, em cães com idade superior a dois anos e, durante esse longo período de parasitismo, a maioria dos cães permanece assintomático devido à compensação das funções pelo rim não parasitado. Outros manifestam sinais como hematúria, dor na região lombar, ou o parasitismo pode ser um achado incidental em procedimentos cirúrgicos ou de necropsia (Pedrassani et al., 2010; 2017).

A progressão da dioctofimose resulta na destruição total do parênquima renal, sendo substituído pelo parasito envolvido pela cápsula fibrosa do órgão (Silveira et al., 2015). No entanto, geralmente não há perda da função renal pela hipertrofia compensatória do rim não parasitado (Monteiro et al. 2002; Pedrozo et al., 2013; Sapin et al., 2017), o que explica a não significância no resultado da mensuração da ureia sanguínea nos animais pesquisados $(\mathrm{p}=0,712)$. Dibartola (2004) cita que a mensuração dos níveis séricos de ureia não é tão fidedigna quanto da creatinina. Seu nível sanguíneo pode estar diminuído devido a outros fatores como dieta baixa em proteína ou insuficiência hepática grave como poder estar aumentado por um jejum prolongado antes da coleta de sangue.

Em relação à creatinina sérica o resultado mostrou que em cães parasitados estava aumentada quando comparado aos não parasitados. Visto que a creatinina não sofre influência de outros fatores e é excretada quase inteiramente pela filtração glomerular, sua mensuração é importante na pesquisa de disfunção renal já que uma vez aumentada, significa danos renais (Dibartola, 2004). A observação de cães parasitados e com níveis de creatinina sérica dentro dos valores normais pode ser explicado pelo fato de que geralmente o rim contralateral tenta compensar o rim afetado (Measures, 2001). Cottar et al. (2012) citam que algumas vezes o rim não parasitado não consegue compensar e manter a função renal, o que pode justificar as alterações de creatinina observadas nos cães parasitados.

Anemia não foi detectada pelo hemograma dos animais analisados neste trabalho e Measures e Anderson (1985) afirmam que a anemia pode ser uma manifestação clínica da dioctofimose, mas nem sempre está presente, pois não é um sinal patognomônico. O parasitismo provavelmente tem a capacidade de levar a anemia devido à destruição que o parasito promove no parênquima renal, comprometendo a produção de eritropoietina pelos rins (importante hormônio que participa da eritropoese) (Nelson; Couto, 2015).

Neste estudo, a hiperproteinemia detectada nos dois grupos pode ser em decorrência da desidratação dos cães e/ou da hemólise nas amostras, que podem ter contribuído para o aumento da proteína plasmática total (PPT) (Bush, 2004). Grant e Forrester (2008), afirmam que a hiperproteinemia nos exames pode ser um 
indício de dioctofimose. Durante a resposta aguda na dioctofimose canina, Schmidt et al. (2016) observaram um aumento na concentração de haptoglobina (proteína da fase aguda de processos inflamatórios) o que pode ter contribuído na hiperproteinemia dos cães parasitados.

Os dados dessa pesquisa demonstraram, pela primeira vez, a presença de parasitismo por $D$. renale em cães do município de São Mateus do Sul, Paraná vivendo em condições favoráveis à infecção. Como os animais examinados eram de abrigos de ONG, existe a possibilidade de serem oriundos de outros municípios e terem adquirido a infecção nesses outros locais. No município de Três Barras, pertencente ao Estado de Santa Catarina, mas que faz divisa com São Mateus do Sul, já foram relatados casos de parasitismo, por formas adultas em cães e por formas larvais em sapos (Pedrassani et al., 2010; 2017). Por ser zoonótico o ser humano deve ter cuidados em relação à ingestão de água, que deve ser de fonte conhecida e tratada e à ingestão de peixes de água doce crus ou mal assados (Yang et al., 2019) oriundos da região.

O exame do sedimento urinário pode ser utilizado como uma ferramenta auxiliar para a detecção precoce de casos de dioctofimose nos animais. É importante lembrar que nos casos de infecções somente com nematódeos machos, parasitos imaturos ou em localizações ectópicas, o exame não será capaz de detectar o parasitismo, resultando em diagnóstico falso negativo (Pedrassani et al., 2017).

\section{CONCLUSÃO}

Exames rotineiros de urinálise são relevantes para um diagnóstico precoce de parasitismo por Dioctophyme renale. A urinálise permitiu a detecção de ovos de $D$. renale em cães assintomáticos procedentes de abrigos de uma ONG. Porém, esse método pode apresentar resultados falso-negativos, portanto, exames complementares podem ser necessários.

O diagnóstico é importante, pois cães parasitados servem como fontes de infecção para hospedeiros intermediários e hospedeiros paratênicos que vivem na região podendo expor em risco a saúde humana.

\section{NOTA INFORMATIVA}

Todos os procedimentos utilizados foram aprovados pela Comissão de ética no Uso e Experimentação de Animais - CEUA da Universidade do Contestado (certificado $\mathrm{n}^{\circ}$ 07/18).

\section{AGRADECIMENTOS}

Os autores agradecem a Universidade do Contestado, por meio do Fundo de Apoio à Pesquisa (FAP), pelo auxílio financeiro.

\section{REFERÊNCIAS}

BUSH, B. M. Interpretação de resultados laboratoriais para clínicos de pequenos animais. São Paulo: Roca, 2004.

COLPO, C. B.; SILVA, A. S.; MONTEIRO, S. G.; STAINKI, D. R.; CAMARGO, D. G.; COLPO, E. T. B. Ocorrência de Dioctophyma renale em cães no Município de Uruguaiana - RS. Revista da Faculdade de Zootecnia, Veterinária e Agronomia, Uruguaiana, v. 14, n. 2, p. 175-180, 2007. 
COTTAR, B. H.; DITTRICH, G.; FERREIRA, A. A. CARVALHO, A. C. P.; ALBERNAZ, V. G. P.; LUZ, M. T.; TASQUETI, U. I. Achados ultrassonográficos de cães parasitados por Dioctophyma renale: estudo retrospectivo. Veterinária e Zootecnia, v.19, n. 1, p. 8-11, 2012.

DIBARTOLA, S. P. Abordagem clínica e avaliação laboratorial da doença renal. In: ETTINGER, J.S.; FELDMAN, E.C. Tratado de Medicina Interna Veterinária doença do cão e do gato. Rio de Janeiro: Guanabara Koogan, 2004, p. 1686-1700.

GIORELLO, A.N.; KENNEDY, M. W.; BUTTI, M. J.; RANDAN, N. E.; CÓRSIO, B.; FRANCHINI, G. R. Identification and characterization of the major pseudocoelomic proteins of the giant kidney worm, Dioctophyme renale. Parasites \& Vectors, v. 10, n 446, p 1 - 11, 2017.

GRANT, D.; FORRESTER, S.D. Doenças do sistema urogenital, doenças do rim e ureter. In: BICHARD, S.J.; SHERDING, R.G. Manual Saunders: Clínica de Pequenos Animais. São Paulo: Roca, 2008, p.882-909.

Instituto Brasileiro de Geografia e Estatística. IBGE, 2018. Cidades: São Mateus do Sul. Disponível em: <https://www.ibge.gov.br/cidades-e-estados/pr/sao-mateus-dosul.html>. Acesso em: 10 nov. 2018.

KANO, F. S.; SHIMADA, M. T; SUZUKI, S. N.; OSAK, S. C.; MENARIM, B. C.; RUTHES, F.R V.; FILHO, M. A. L. Ocorrência da dioctofimose em dois cães no município de Guarapuava - PR. Semina: Ciências Agrárias, Londrina, v. 24, n. 1, p. 177-180. 2003.

KERR, M. G. Exames Laboratoriais em Medicina Veterinária - Bioquímica Clínica e Hematologia. 2. Ed., São Paulo: Roca, 2003, p. 421.

KOMMERS G. D.; ILHA, M. R. S.; BARROS, C. S. L. Dioctofimose em cães: 16 casos. Ciência Rural, v.29, n.3, p.517-522, 1999.

LI, G.; LIU, C.; LI, F.; ZHOU, M.; LIU, X.; NIU, Y. Fatal bilateral dioctophymatosis. The Journal of Parasitology. v.96, p.1152-1154, 2010.

LIMA, C. S.; MURAKAMI, V.; NAKASU, C. C. T; MILECH, V.; DURANTE, L. H.; PERERA, S. C.; CLEFF, M. B.; RAPPETI. J. C. S; CRIVELLENTTI, L. Z. Dioctophyme renale o verme gigante do rim: revisão de literatura. Revista Investigação, Franca, n. 4, p. 37-41, 2016.

MASCARENHAS, C. S.; MULLER, G.; MACEDO, M. R. P; HENZEL, A. B.D.; RABALDO, R. B.; CORREA, F. The role of freshwater fish in the life cycle of Dioctophyme renale in Southern Brazil. Veterinary Parasitology: Regional Studies and Reports, v. 16, p.1-7, abr. 2019.

MEASURES, L. N. Dioctophymatosis. In: SAMUEL, W. M., PYBUS, M. J., KOCAN, A. A. Parasitic diseases of wild mammals. 2. Ed. lowa: lowa State University Press; 2001. p. 357-364. 
MEASURES, L.N.; ANDERSON R. Centrarchid fish as paratenic hosts of the giant kidney worm, Dioctophyme renale in Ontario, Canadá. Journal of Wildlife Disease. v. 21, n.1, p.11-19. Jan. 1985.

MONTEIRO, S.G.; SALLIS, E. S.; STANKI, D. R. Infecção natural por trinta e quatro helmintos da espécie Dioctophyma renale (GOEZE, 1782) em um cão. Revista da FZVA, Uruguaiana. v.9, n.1, p.95-99. 2002.

NELSON, R. W.; COUTO, C. G. Medicina Interna de Pequenos Animais. 5. Ed. Rio de Janeiro: Elsevier, 2015.

PEDRASSANI, D.; WENDT, H.; RENNAU, E. A; PEREIRA, S. T.; WENDT, S. B. T. Dioctophyme renale Goeze, 1782 in a cat with a supernumerary kidney. Brazilian Journal of Veterinary Parasitology, Jaboticabal, v. 23, n. 1, p. 109-117, Jan-Mar 2014.

PEDRASSANI D.; HOPPE, E. G. L.; TEBALDI, J. H.; NASCIMENTO, A. A. Chaunus ictericus (Spix, 1824) as paratenic host of the giant kidney worm Dioctophyme renale (Goeze, 1782) (Nematoda: Enoplida) in São Cristóvão district, Três Barras county, Santa Catarina state, Brazil. Veterinary Parasitology, v. 165, n. 1-2, p. 74-77, 2009.

PEDRASSANI, D.; PILATI, C.; WENDT, S. B. T.; MACHADO, R. Z.; NASCIMENTO, A.A. Diagnóstico ultrassonográfico de infecção intensa por Dioctophyme renale em rim esquerdo de cão - relato de caso. Clínica Veterinária, São Paulo, n.85, p. 74 80, 2010.

PEDRASSANI, D.; NASCIMENTO, A. A.; ANDRE, M. R.; MACHADO, R. Z. Dioctophyme renale: prevalência e fatores de risco de parasitismo em cães no distrito de São Cristóvão, Três Barras, Santa Catarina, Brasil. Brazilian Journal of Veterinary Parasitology, Jaboticabal, v. 26, p. 39 - 46, Fev. 2017.

PEDROZO, R.; BRAZAN, A.; CUEVAS, D.; GODOY, Y. Valores séricos de urea, creatinina y densidad urinaria en perros com Dioctophyma renale en La ciudad Caazapá, Paraguay, y surelación com factores de riesgo, Compêndio de CiênciasVeterinárias, v. 3, n.2, p.33-38, Dez.2013.

PEREIRA, M. G. Epidemiologia: Teoria e prática, 12. Ed., Rio de Janeiro: Guanabara Koogan, 2008.

PERERA, S. C.; RAPPETI, J. C. S; MILECH, V.; BRAGA, F. A.; CAVALCANTI, G. A. O.; NAKASU, C. C.; DURANTE, L.; VIVES, P.; CLEFF, M. B. Eliminação de Dioctophyme renale pela urina em canino com dioctofimatose em rim esquerdo e cavidade abdominal - Primeiro relato no Rio Grande do Sul. Arquivo Brasileiro de Medicina Veterinária e Zootecnia, Belo Horizonte, v. 69, n. 3, p. 618-622, Jun. 2017.

RAPPETI, J. C. S.; MASCARENHAS, C. S.; PERERA, S. C.; MULLER, G.; GRECCO, F. B.; SILVA, L. M. C.; SAPIN, C. F.; RAUSCH, S. F.; CLEFF, M. B. Dioctophyme renale (Nematoda: Enoplida) in domestic dogs and cats in the extreme south of Brazil. Revista Brasileira de Parasitologia Veterinária, Jaboticabal, v. 26, p 119-121, Jan./Mar, 2017. 
ROCHA, M. F. Dioctophyma renale em testículo de cão no município de Curitibanos, SC, Brasil - Relato de Caso. 2017. Disponível em: <https://repositorio.ufsc.br/xmlui/bitstream/handle/123456789/182390/Monografia_M arilise.pdf?sequence=1\&isAllowed=y>. Acesso em: 05 jul. 2019.

SAPIN, C. F., SILVA-MARIANO, L. C., GRECCO-CORRÊA, L., RAPPETI, J. C.S., DURANTE, L. H., PERERA, S. C., CLEFF, M. B., GRECCO, F. B. Dioctofimatose renal bilateral e disseminada em cão. Pesquisa Veterinária Brasileira, v. 37, n. 1), p. 1499-1504, 2017.

SCHMIDT, E.M.S.; KJELGAARD-HANSEN, M.; THOMAS, F.; TVARIJONAVICIUTE, A.; CÉRON, J. J; ECKERSALL, P. D. Acute phase proteins in dogs naturally infected with the Giant Kidney Worm (Dioctophyme renale), Veterinary Clinical Pathology, v.45, n.4, p.657-664, 2016.

Secretaria de Estado do Meio Ambiente e Recursos Hídricos. SEMA. Bacias Hidrográficas do Paraná - Série Histórica, Curitiba, 2010.

SECCHI P.; VALLE, S. F.; BRUN, M. V.; MOTTA, A, C.; RAUSCH, S. F.; MESSINA, S. A.; VIEIRA, M. I. B . Nefrectomia videolaparoscópica para tratamento da dioctofimose em um cão. Acta Scientiae Veterinariae, Porto Alegre, v. 38, n.1, p. 85-89, 2010.

SILVEIRA, C. S.; DIEFENBACH, A.; MISTIERI, M. L.; MACHADO, I. R. L.; ANJOS, B. L. Dioctophyma renale em 28 cães: aspectos clinico patológicos e ultrassonográficos. Pesquisa Veterinária Brasileira, Rio de Janeiro, v. 35, n. 11, p.899-905, nov. 2015.

SINK, C.A.; FELDMAN, B.F. Urinálise e Hematologia Laboratorial para o Clínico de Pequenos Animais. São Paulo: Roca, 2006.

SLOSS, M.W.; ZAJAC, A.M.; KEMP, R.L. Parasitologia clínica veterinária. São Paulo: Manole; 1999.

SOUZA, M. S.; M. S.; DUARTE, G. D.; BRITO, S. A. P.; FARIAS, L. A.. Dioctophyma renale: revisão. Pubvet: Medicina Veterinária e Zootecnia, João Pessoa. v.13, n.6, a346, p.1-6, Jun., 2019.

WEISER, G. Princípios gerais de exames e diagnósticos laboratoriais. In: THRALL, M. A. Hematologia e bioquímica - Clínica veterinária. Rio de Janeiro: Roca, 2015. p. $1-49$.

YANG, F.; ZHANG, W.; GONG, B.; YAO, L.; LIU, A.; LING, H. A human case of Dioctophyma renale (giant kidney worm) accompanied by renal cancer and a retrospective study of dioctophymiasis. Parasite, v. 26, n. 22, p. 1-8, 2019. 